\title{
EFFECT OF TEMPERATURE AND GERMINATION MEDIA ON A VIABILITY OF POLLEN GRAIN OF FIVE PISTACHIO MALE STRAINS
}

Mohamad Kardoush*, Mohamad Ayman Dairy*,.Saleh Shdeifat**, Naif Albashabsheh***

* Depart of Horti- Fac of Agriculture Univ of Aleppo. ** Ministry of Agriculture Jordan. $* * *$ Postgraduate Student $(\mathrm{PhD})$.

\begin{abstract}
This experiment was conducted to evaluate the effect of germination media and three temperatures on five male strains of pistachio at research center of agriculture faculty - Aleppo,(Arab center),.In this experiment, the rates of pollen germination were tested in different germination media (sucrose 10\%, sucrose $10 \%+0.25$ gr. boric acid P L. and sucrose $10 \%+0.0625$ gr. boric acid P L.) with 3 temperature levels( 10,20 and $30^{\circ} \mathrm{c}$ ). The media was contain 3 gr. agar per liter of water. The results showed that male strain 36 was gave the best germination rate, (78.33\% and tube length 796.1micron) as compared with other strains ,and male strain 47 was the lower $(63.11 \%, 511.3$ micron) tube germination and tube length (63.11\%,511.3 micron) respectively, but all were gave high rates of germination. sucrose $10 \%$ media gave the best germination rate $(77.07 \%)$ and sucrose $10 \%+$ 0.25 gr. boric acid P L. gave the lowest germination rate (62.2\%), sucrose $10 \%+0$. 0.25 gr. boric acid $\mathrm{P}$ L. gave highest tube germination length (777.68micron), where as $10 \%$ sucrose +0.0625 gr. boric acid P L. was the lowest tube length (499.88micron). On the other hand, $30^{\circ} \mathrm{c}$ gave the best germination rate and pollen tube germination length $\left(88.73 \%\right.$ and 1005.26 micron), however $10^{\circ} \mathrm{c}$ gave lowest rates of these parameters $(45.53 \%$ and, 244.3 micron) for germination rate and tube pollen length respectively. Generally, all media were gave highest significant results in germination rates and pollen tube length for all strains at all temperatures. we conclude that sucrose $10 \%$ was better media for pollen germination and sucrose $10 \%+0.25$ gr. boric acid P L.was the best for pollen tube length .
\end{abstract}

\section{INTRODUCTION}

Pistachio trees are dioecious with male and female flowers on separate trees. Male and female trees must be present for cross pollination and fruit set. In the Arab Center Farm for studies of Arid Zone and dry lands at Aleppo ,there are a lot of different male strains used as a pollinators of pistachio trees. It is necessary to know a viability and germination rate of pollen grain before pollination date to be able to ensure fruit set(Dafni\&Firmage,2000). There are many studies published about germination media and temperature in recent years .Sfendiaro et al., (2006) reported that pollen viability in TTC test was ranged between 85.7 and $98.85 \%$ in different male types of pistachio. 
Hadj-Hassan (1985) was arranged male types of pistachio according to germination rate from weak to excellent germination (less than $25 \%$ weak,26- 40 $\%$ medial, $41-60 \%$ good and highest than $61 \%$ excellent viability.

Acar and AK (2007) Reported that the highest germination rate of pistachio pollen was obtained from sucrose $20 \%$ (87.78\%), and they found that adding $\mathrm{H}_{3} \mathrm{BO}_{3} \quad 20$ and $70 \mathrm{ppm}$ germ ination rate obtained over 80\%.Ulkumen (1945) reported that the highest germination rate was obtained from 10 and $15 \%$ sucrose solution in Pistacia vera, $P$. terebinthus, $P$. atlantica. Therios et al(1985) showed that maximum germination obtained at 35,20 and $25 \%$ sucrose for the clones A, B and $\mathrm{C}$ of pistachio male strains respectively.

This experiment shows that sucrose concentrations may change from male type to another type. According to Atli et al(1995)The highest pollen germination rate of pistachio was observed by using $15 \%$ sucrose solution in tube method, and $10 \%$ sucrose solution in Petri dish method. Crane et al(1974) reported that the highest germination rate obtaind was $94 \%$ by using $10 \%$ sucrose solution at peters pollen, Beside that, $72 \%$ germination rate was obtained by using $15 \%$ sucrose solution. AK et al(1995)reported that the best pollen germination rate was obtained by using 10-15\% sucrose solution .Mlika(1991) mentioned that the pollen germination rate was obtained in pistachios ranged from $57-59 \%$ by using 10 $20 \%$ sucrose solution.Cagler \&Kaska(2007)reported that germination rate of pistachio pollen was ranged between 55-69\%using $15 \%$ sucrose solution for many male types.

The aim of this study was to investigate the pollen germination rate and germination of tube length of pistachio male trees at different media and temperatures becuase there were no studies about pollen tube length in Arab Center Farm .at Aleppo-Syria.

\section{MATERIAL AND METHODS}

In this experiment 5 male strains of pistachio(36, 38, 42, 47, and57) grown in pistachio orchard at Arab center farm in Aleppo were used as material.Pollen collection: The branches of selected male types were taken to the laboratory conditions $(20 \mathrm{c})$ before the cluster dehisced pollen, and they were put in glass container filled with water. After waiting one night, the pollen had been taken and sowed with brush to the different germination media.

The germination media:10\% Sucrose, $10 \%$ sucrose+0.25gr. boric acid P L. media and $10 \%$ sucrose +0.0625 gr. boric acid P L. media , adding 3 g agar per 1 liter toeach media .

The germination temperatures were: 10,20 and $30^{\circ} \mathrm{c}$. The experimental design: we used factorial design with 3 factors: (5 male strains, 3 media and 3 temperature). arranged in 3 replications with M-Stat .C. Program. Germination rate was recorded by 
Eq: G.R = \#Gpollen $\backslash \#$ of total pollen ${ }_{x} 100$, and pollen tube length/micron.by using scale lens Fig (5).

The pollen grain counting and pollen tube length were performed after 24 hours in incubation at 3 temperatures .fig (1-5)

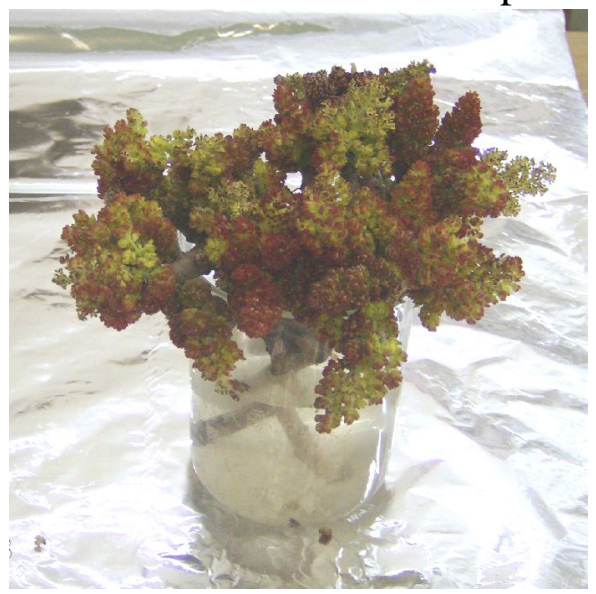

Figure1: pollen grain collection

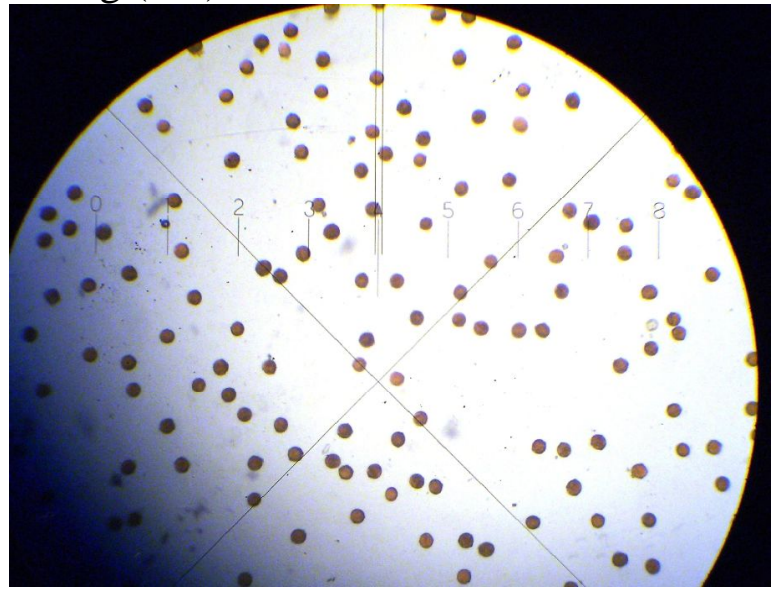

Figure2: pollen grain before germination

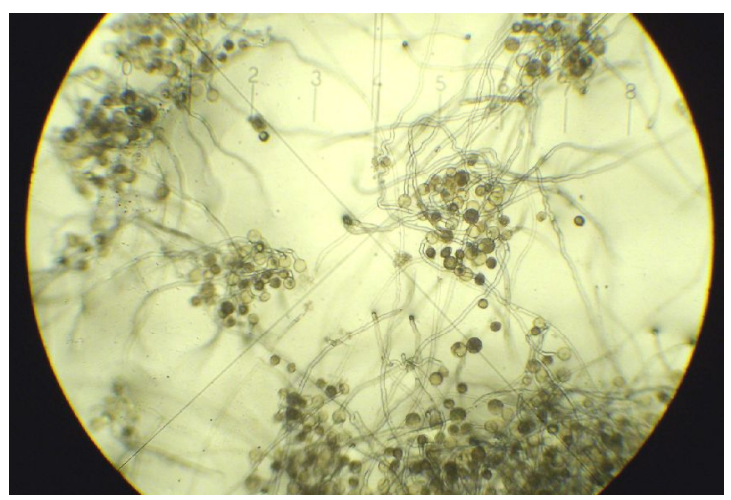

Figure 3:pollen germination and pollen at $20^{\circ} \mathrm{C}$

length at tube $30^{\circ} \mathrm{c}$

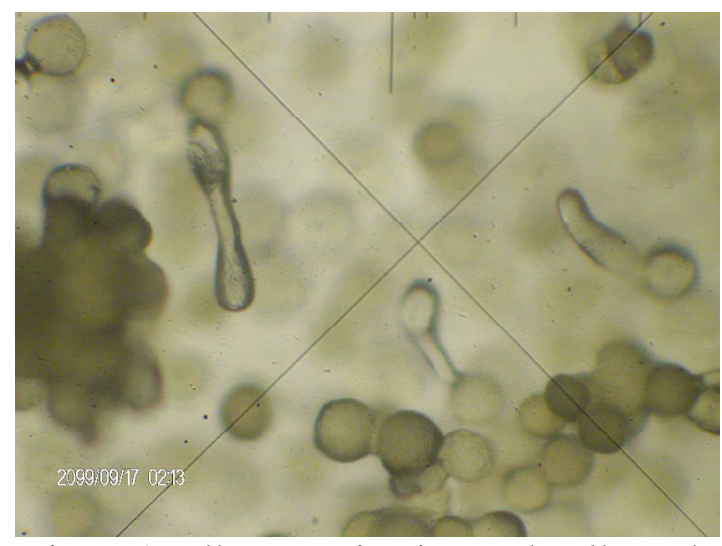

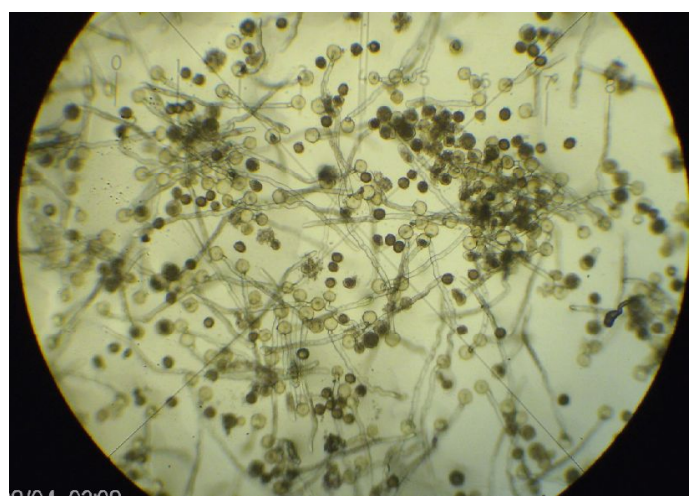

Figure 4:pollen germination and tube length

Figure5:pollen germination and pollen tube length at $10 \AA \mathrm{c}$ 


\section{RESULTS AND DISCUSSION}

Effect of different germination media on the germination rate of selected male strains at Arab center orchard were given in table 1.

The pollen germination rate was better in male No.36 $(78.33 \%)$ than the other male types, however male No.47 was the lowest $(63.11 \%)$.The germination rate of males ranged between $63.11 \%-78.33 \%$.

Table 2 :indicate the effect of different germination media on the length of pollen tube of selected male strain at Arab center orchard .

Table(1): Effect of different media on germination rate\% of pistachio male types at 2008 season.

\begin{tabular}{|c|c|c|c|c|}
\hline Strain\media & $10 \%$ sucrose & $\begin{array}{l}10 \% \text { sucrose } \\
0.25 \text { gr boric }\end{array}$ & $\begin{array}{c}10 \% \text { sucrose }+0 \\
0625 \text { gr. boric }\end{array}$ & mean \\
\hline 36 & $85 \mathrm{ab}$ & $63.33 \mathrm{~h}$ & $86.67 \quad \mathrm{a}$ & $78.33 \mathrm{a}$ \\
\hline 38 & $83.33 \mathrm{~b}$ & $71.67 \mathrm{fg}$ & 75 de & $76.67 \mathrm{~b}$ \\
\hline 42 & $80.33 \mathrm{c}$ & $70 \quad \mathrm{~g}$ & $76.67 \quad \mathrm{~d}$ & $75.67 \mathrm{~b}$ \\
\hline 47 & $63.33 \mathrm{~h}$ & $54.33 \quad \mathrm{i}$ & 71.67 & $63.11 \mathrm{~d}$ \\
\hline 57 & 73.33 ef & 51.67 & 73.33 & $66.11 \mathrm{c}$ \\
\hline mean & $77.07 \mathrm{a}$ & $62.2 \mathrm{c}$ & 76.67 & \\
\hline Treat. & \multicolumn{2}{|c|}{ L.S.D 0.01} & \multirow{4}{*}{\multicolumn{2}{|c|}{$\begin{array}{c}\% \mathrm{CV} \\
5.65\end{array}$}} \\
\hline Strain & \multicolumn{2}{|c|}{1.559} & & \\
\hline Media & \multirow{2}{*}{\multicolumn{2}{|c|}{1.208}} & & \\
\hline Media x strain & & 2.701 & & \\
\hline
\end{tabular}

Results indicated that $10 \%$ sucrose was better media in germination rate $(77.07 \%)$, where as

$10 \%$ sucrose+0.25gr. boric acid P L gave the lowest germination rate $(62.2 \%)$ related to toxic effect of boric acid at high concentration .

Table(2): Effect of different media on pollen tube length (micron) of five pistachio male types at 2008 season.

\begin{tabular}{|c|c|c|c|c|}
\hline Strain \media & $10 \%$ sucrose & $\begin{array}{r}10 \% \text { sucrose+ } \\
0.25 \text { gr boric } \\
\end{array}$ & $\begin{array}{l}10 \% \text { sucrose }+0.0625 \mathrm{gr} . \\
\text { boric }\end{array}$ & mean \\
\hline 36 & $80^{\circ} .3 \quad \mathrm{c}$ & $102^{\wedge} \quad \mathrm{a}$ & $555.3 \mathrm{~g}$ & $796.1 \quad \mathrm{a}$ \\
\hline 38 & 750 & 805.4 & Tr人.V & $\begin{array}{c}731.3 \\
\mathrm{~b}\end{array}$ \\
\hline 42 & 611 & 638.7 & 527.7 & $\begin{array}{c}592.47 \\
\mathrm{~d}\end{array}$ \\
\hline 47 & $395.3 \quad \mathrm{j}$ & $861.0 \quad \mathrm{~b}$ & $277.7 \mathrm{k}$ & $511.3 \mathrm{e}$ \\
\hline 57 & $861.0 \quad \mathrm{~b}$ & $555.3 \mathrm{~g}$ & $500.0 \quad \mathrm{i}$ & 638.8 \\
\hline Mean & $684.5 \quad b$ & $777.68 \quad \mathrm{a}$ & $499.88 \mathrm{c}$ & \\
\hline Trt & & L.S.D $1 \%$ & \multirow{4}{*}{$\begin{array}{l}\% \mathrm{CV} \\
3.69\end{array}$} & \\
\hline Strain & & 12.83 & & \\
\hline Media & & 9.94 & & \\
\hline mediax strain & & 22.23 & & \\
\hline
\end{tabular}


Male No.36 was the better in pollen tube length (796.1 micron) than other Strain , and male No.47gave the lowest tube length (511.3 micron) .The lengths of pollen tube were arranged from 511.3 to 796.1 micron.

The results of this study indicated ,that the length of pollen tube germination was increased with increasing of boric acid concentrations .

Table 3 indicate the effect of different temperatures on germination rate of selected pistachio male types .

Table(3): Effect of different temperatures on germination rate $\%$ of pistachio male types at 2008 season.

\begin{tabular}{|c|c|c|c|c|c|}
\hline Strain\temp. & $10^{\circ} \mathrm{C}$ & $20^{\circ} \mathrm{C}$ & $30^{\circ} \mathrm{C}$ & mear & \\
\hline 36 & $65 \quad \mathrm{~g}$ & $78.33 \mathrm{e}$ & 91.67 & 78.33 & $\mathrm{a}$ \\
\hline 38 & $48.33 \mathrm{~h}$ & $88.33 \mathrm{~b}$ & 93.33 & 76.67 & $\mathrm{~b}$ \\
\hline 42 & $48.33 \mathrm{~h}$ & $86.67 \mathrm{bc}$ & 92 & 75.67 & $\mathrm{~b}$ \\
\hline 47 & $21 \mathrm{j}$ & $85 \mathrm{~cd}$ & $83.33 \mathrm{c}$ & 63.11 & $\mathrm{~d}$ \\
\hline 57 & $45 \mathrm{i}$ & $70 \mathrm{f}$ & $83.33 \mathrm{c}$ & 66.11 & $\mathrm{c}$ \\
\hline Mean & $45.53 \mathrm{c}$ & $81.67 \mathrm{~b}$ & 88.73 & \multirow{5}{*}{\multicolumn{2}{|c|}{$\begin{array}{c}\% \mathrm{CV} \\
5.65\end{array}$}} \\
\hline Trt & \multicolumn{3}{|c|}{$1 \%$ LSD } & & \\
\hline Strain & \multicolumn{3}{|c|}{1.559} & & \\
\hline temperature & \multicolumn{3}{|c|}{1.432} & & \\
\hline temperature $\mathrm{x}$ strain & \multicolumn{3}{|c|}{3.202} & & \\
\hline
\end{tabular}

Male No.36 gave the highest germination rate than others $(78.33 \%)$ where as the male No.47 gave the lowest germination rate than other strain $(63.11 \%)$ .The germination rate were ranged between 63.11 and $78.33 \%$. The temperature $30^{\circ} \mathrm{C}$ showed the highest germination rate $(88.73 \%)$ compared to other temperatures, where as temperature $10^{\circ} \mathrm{c}$ gave the lowest germination rate $(45.53 \%)$ . We observed that the germination rate was increased with temperature increasing .We observed that the high temperature $(30 \mathrm{c})$ was a suitable to germination of pollen grain.

Table 4 showed the effect of different temperatures on pollen tube length of selected types of pistachio males at. $30^{\circ} \mathrm{c}$ temperature.

The temperature at $30^{\circ} \mathrm{c}$ gave the highest pollen tube length (1005.26 micron) when compared to other strains, where as the $10^{\circ} \mathrm{c}$ gave the lowest tube length (244.3 micron). Male No.36 gave the highest tube length (796.1 micron) ,however the male No. 47 gave the lowest tube length (511.3 micron) than other strains .These results indicate the role of genetic variation between male types and high temperature in increasing pollen tube length as we mentioned .

Table(4): Effect of different temperatures on pollen tube length( micron) of five pistachio male types at 2008 season.

\begin{tabular}{|c|c|c|c|c|}
\hline Strain\temp. & $100^{\circ} \mathrm{C}$ & $200^{\circ} \mathrm{C}$ & $30^{\circ} \mathrm{C}$ & Mean \\
\hline 36 & $527.7 \quad \mathrm{j}$ & $916.7 \mathrm{e}$ & $944 \quad \mathrm{~d}$ & $796.1 \quad a$ \\
\hline 38 & 222.0 & 805.7 & 1166. a & 731.3 \\
\hline 42 & 222.3 & $499.7 \mathrm{k}$ & $1055 \mathrm{~b}$ & $592.47 \mathrm{~d}$ \\
\hline 47 & $111 \mathrm{n}$ & $589.7 \mathrm{i}$ & $833.3 \mathrm{f}$ & 511.3 \\
\hline
\end{tabular}


2009

\begin{tabular}{|c|c|c|c|c|}
\hline 57 & $138.7 \mathrm{~m}$ & $750 \mathrm{~h}$ & $1028 \mathrm{c}$ & 638.8 \\
\hline Mean & $244.3 \mathrm{c}$ & $714.16 \mathrm{~b}$ & $1005.26 \mathrm{a}$ & \\
\hline Trt & \multicolumn{2}{|c|}{ LSD $1 \%$} & \multirow{4}{*}{$\begin{array}{c}\% \mathrm{CV} \\
3.69\end{array}$} & \\
\hline Strain & \multicolumn{2}{|c|}{12.83} & & \\
\hline temperature & \multicolumn{2}{|c|}{8.495} & & \\
\hline Temperature x strain & \multicolumn{2}{|c|}{19} & & \\
\hline
\end{tabular}

Table 5 explain the effect of interaction among media, temperature and strain on germination rate of five selected male types of pistachio at Arab center orchard at Aleppo.

The results in table5 showed that there were a significant different between interaction treatments. Male No.36grown on $10 \%$ sucrose media at $20^{\circ} \mathrm{c}$ temperature gave the highest germination rate (100\%), whereas Male No. 47 at $10^{\circ} \mathrm{c}$ temperature with $10 \%$ sucrose +0.0625 gr. boric acid p L media gave the lowest germination rate $(3 \%)$.

The germination rate of males was affected by interaction between male types $\mathrm{X}$ media $\mathrm{X}$ temperature which were ranged between $63.11 \%$ to $78.33 \%$.

Table 6 showed the effect of interaction among media $\mathrm{X}$ temperature and strain on pollen tube length of selected male types of pistachio.The male No.47 pollen tube grown in $\left(10 \%\right.$ sucrose $+0.25 \mathrm{gr}$. boric acid at $30^{\circ} \mathrm{c}$ temperature gave the highest pollen tube length (1500micron) with a significant differences than other treatments, where as this male No. 47 grown with the same media at $10^{\circ} \mathrm{c}$ temperature gave the lowest pollen tube length (83micron). That mean their are differences between males at different temperatures and different media. The length of pollen tube arranged from 511.3 to 796.1. We observed that the high length of pollen tube in $10 \%$ sucrose $+0.25 \mathrm{gr}$. boric acid P L media , that mean the increasing of boric acid concentration caused an increase in tube length .

Results indicated that male strain types of pistachio was differ in germination rate and pollen tube length that means, male types of pistachio was differ in germination rate,this variation may be related to the genetic features ,these results agree with (Hadj-Hassan,1985). On the other hand 10\% Sucrose media gave better result than other media (Acar \&AK,2007), and this result in accordance with (Johri and Vasil,1961), (Crane et al,1974). The adding of boric acid to media gave good result at low concentration, and this result agree with (Therios et al, 1985; AK,et al,1995). The adding of boric acid to media gave better result in germination rate at low concentration, however the pollen tube length was the highest in $10 \%$ sucrose $+0.0625 \mathrm{gr}$. boric acid media, and this result agree with (Therios et al,1985;AK,et al,1995). This study showed that the media concentration was changed from male type to other. We observed that the germination rate and the pollen length increased when temperature increased because temperature at $30 \mathrm{c}$ promote germination of pollen . 


\section{CONCLUSION}

Using of $10 \%$ Sucrose media at $20-30^{\circ} \mathrm{c}$ for pollen germination and $10 \%$ sucrose +0 . $25 \mathrm{gr}$. boric acid $\mathrm{PL}$ at $30^{\circ} \mathrm{c}$ to increasing tube length, and gave the best results .

Tble(5): Effect of interaction among media, temperature and strain on germination rate $\%$ of five pistachio male types in 2008 season.

\begin{tabular}{|c|c|c|c|c|c|}
\hline \multirow[t]{2}{*}{ Strain } & \multirow[t]{2}{*}{ Temp. } & \multicolumn{3}{|c|}{ Media } & \multirow[t]{2}{*}{ mean } \\
\hline & & $10 \%$ sucrose & $\begin{array}{c}10 \% \text { sucrose } \\
+0.25 \text { gr. boric }\end{array}$ & $\begin{array}{c}10 \% \text { sucrose }+0.0625 \mathrm{gr} . \\
\text { boric acid }\end{array}$ & \\
\hline \multirow[t]{3}{*}{36} & 10 & $95 \mathrm{ab}$ & $10 \quad \mathrm{~m}$ & $90 \quad$ bc & \multirow{3}{*}{$78.33 \mathrm{a}$} \\
\hline & 20 & $100 \mathrm{a}$ & $85 \mathrm{~cd}$ & 90 bc & \\
\hline & 30 & 60 hi & $80 \mathrm{de}$ & $95 \mathrm{ab}$ & \\
\hline \multirow[t]{3}{*}{38} & 10 & $60 \mathrm{hi}$ & $30 \quad 1$ & $55 \quad \mathrm{ij}$ & \multirow{3}{*}{$76.67 \mathrm{~b}$} \\
\hline & 20 & $95 \mathrm{ab}$ & 90 bc & $80 \mathrm{de}$ & \\
\hline & 30 & $95 \mathrm{ab}$ & $95 \mathrm{ab}$ & $90 \mathrm{bc}$ & \\
\hline \multirow[t]{3}{*}{42} & 10 & $50 \quad \mathrm{jk}$ & $\begin{array}{ll}30 & 1 \\
\end{array}$ & $65 \mathrm{gh}$ & \multirow{3}{*}{$75.67 \mathrm{~b}$} \\
\hline & 20 & $95 \mathrm{ab}$ & $85 \mathrm{~cd}$ & 80 de & \\
\hline & 30 & 96 a & $90 \mathrm{ab}$ & $85 \mathrm{~cd}$ & \\
\hline \multirow[t]{3}{*}{47} & 10 & 30 ef & $30 \quad 1$ & $3 \quad n$ & \multirow{3}{*}{$63.11 \mathrm{~d}$} \\
\hline & 20 & $85 \mathrm{~cd}$ & 90 bc & $80 \mathrm{de}$ & \\
\hline & 30 & 75 ef & $95 \mathrm{ab}$ & 80 de & \\
\hline \multirow[t]{3}{*}{57} & 10 & 55 ij & $15 \mathrm{~m}$ & $65 \mathrm{gh}$ & \multirow{3}{*}{$66.11 \mathrm{c}$} \\
\hline & 20 & 80 de & 45 & $85 \mathrm{~cd}$ & \\
\hline & 30 & $85 \mathrm{~cd}$ & 70 fg & $95 \mathrm{ab}$ & \\
\hline \multicolumn{2}{|l|}{ Trt. } & LSD $1 \%$ & \multirow{6}{*}{\multicolumn{2}{|c|}{$\begin{array}{l}\text { \% C.V } \\
5.65\end{array}$}} & \\
\hline \multicolumn{2}{|l|}{ Strain } & 1.559 & & & \\
\hline \multicolumn{2}{|l|}{ Media } & 1.208 & & & \\
\hline \multicolumn{2}{|c|}{ Temp. } & 1.432 & & & \\
\hline \multicolumn{2}{|c|}{ Str x med } & 2.701 & & & \\
\hline \multicolumn{2}{|c|}{ Str. X temp } & .3 .202 & & & \\
\hline
\end{tabular}

Table (6): Effect of interaction between media, temperatures and strain on pollen tupe lengt (micron) of pistachio male types in 2008 season.

\begin{tabular}{|c|c|c|c|c|c|}
\hline \multirow[t]{2}{*}{ strain } & \multirow[t]{2}{*}{ Temp. } & \multicolumn{3}{|c|}{ media } & \multirow[t]{2}{*}{ mean } \\
\hline & & $10 \%$ sucrose & $\begin{array}{l}10 \% \text { sucrose }+0.25 \mathrm{gr} . \\
\text { boric }\end{array}$ & $\begin{array}{c}10 \% \text { sucrose }+0.0625 \text { gr. boric } \\
\text { acid }\end{array}$ & \\
\hline \multirow[t]{3}{*}{36} & 10 & $250 \mathrm{k}$ & $1250 \quad \mathrm{c}$ & $83 \quad \mathrm{~m}$ & \multirow{3}{*}{$796.1 \mathrm{a}$} \\
\hline & 20 & 1166. $\mathrm{d}$ & $1083.3 \mathrm{e}$ & $583 \mathrm{~h}$ & \\
\hline & 30 & $1000 \mathrm{f}$ & $1000 \mathrm{f}$ & 750 & \\
\hline
\end{tabular}


Mesopotamia J.of Agric.

(ISSN $1815-316 \mathrm{X})$

Vol.(37) No. (3) 2009

\begin{tabular}{|c|c|c|c|c|c|}
\hline \multirow[t]{3}{*}{38} & 10 & $250 \mathrm{k}$ & $333 \quad \mathrm{j}$ & 83 & \multirow{3}{*}{$731.3 \mathrm{~b}$} \\
\hline & 20 & $1000 \mathrm{f}$ & $1000 \mathrm{f}$ & 417 & \\
\hline & 30 & $1000 \mathrm{f}$ & $1333 \mathrm{~b}$ & $1166 . d$ & \\
\hline \multirow[t]{3}{*}{42} & 10 & $250 \mathrm{k}$ & $250 \mathrm{k}$ & $167 \quad 1$ & \multirow{3}{*}{$\begin{array}{c}592.47 \\
\mathrm{~d}\end{array}$} \\
\hline & 20 & $583 \mathrm{~h}$ & $583 \mathrm{~h}$ & $333 \mathrm{j}$ & \\
\hline & 30 & $1000 \mathrm{f}$ & 1166. d & $1000 \mathrm{f}$ & \\
\hline \multirow[t]{3}{*}{47} & 10 & $\begin{array}{ll}167 \quad 1 \\
\end{array}$ & $83 \mathrm{~m}$ & $83 \mathrm{~m}$ & \multirow{3}{*}{$511.3 \mathrm{e}$} \\
\hline & 20 & $436 \mathrm{i}$ & $333 \mathrm{j}$ & $1000 \mathrm{f}$ & \\
\hline & 30 & $583 \mathrm{~h}$ & $1500 \mathrm{a}$ & $417 \mathrm{i}$ & \\
\hline \multirow[t]{3}{*}{57} & 10 & $250 \mathrm{k}$ & $83 \mathrm{~m}$ & $83 \mathrm{~m}$ & \multirow{3}{*}{$638.8 \mathrm{c}$} \\
\hline & 20 & $1000 \mathrm{f}$ & $1000 \mathrm{f}$ & $250 \mathrm{k}$ & \\
\hline & 30 & $1333 . b$ & 1333. b & $417 \mathrm{i}$ & \\
\hline \multicolumn{2}{|c|}{ Trt. } & LSD $\% 1$ & & \multirow{8}{*}{$\begin{array}{c}\% \text { C.V } \\
3.69\end{array}$} & \\
\hline \multicolumn{2}{|c|}{ Strain } & 12.83 & & & \\
\hline \multicolumn{2}{|c|}{ Media } & 9.94 & & & \\
\hline \multicolumn{2}{|c|}{ Temp. } & 8.495 & & & \\
\hline \multicolumn{2}{|c|}{ Str x med } & 22.23 & & & \\
\hline \multicolumn{2}{|c|}{ Str. X temp } & 19 & & & \\
\hline \multicolumn{2}{|c|}{ Med $x$ temp. } & 14.71 & & & \\
\hline \multicolumn{2}{|c|}{$\begin{array}{l}\text { St. } x \text { med. } x \\
\text { temp. }\end{array}$} & 32.90 & & & \\
\hline
\end{tabular}

تاثير درجات الحرارة واوساط الانبات في نسبة انبات حبوب اللقاح وطول الانبوبة اللقاحيةلخمسة

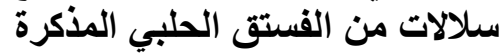

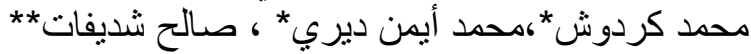

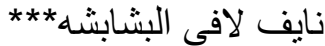

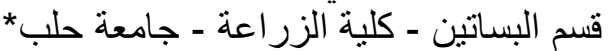

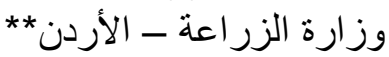

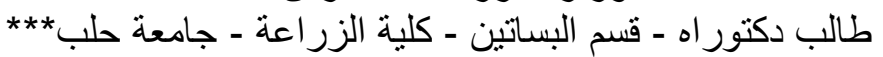

\section{الخلاصة}

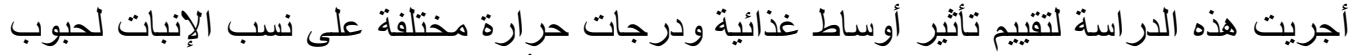

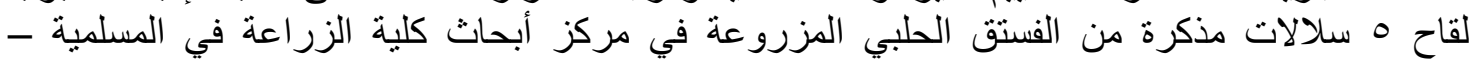

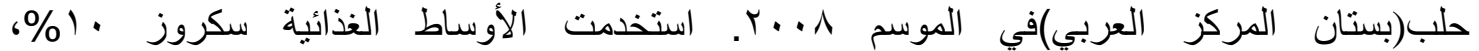

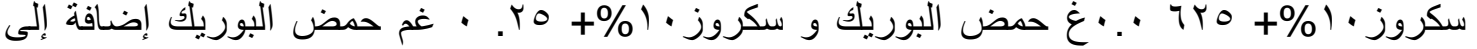

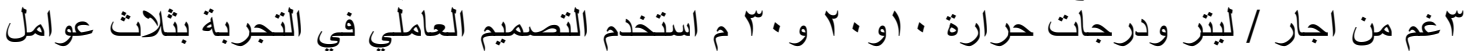

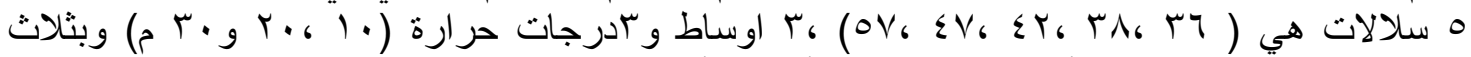

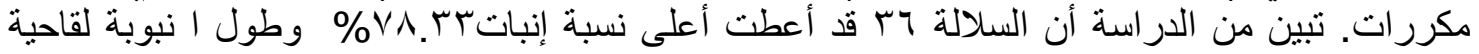

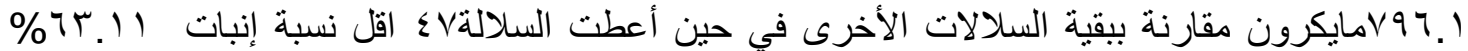

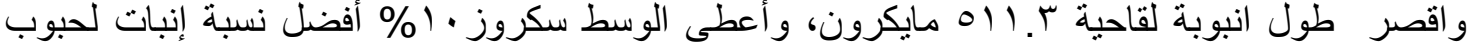

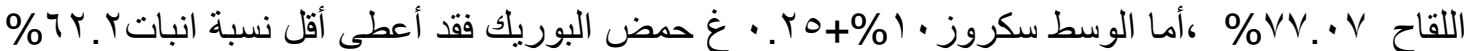

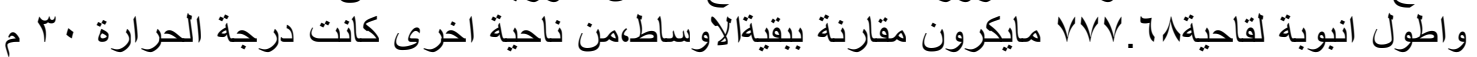

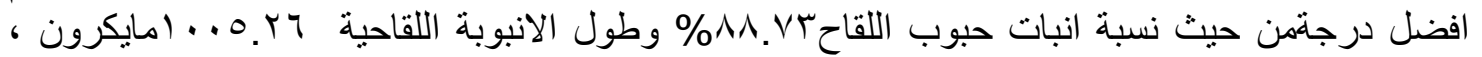




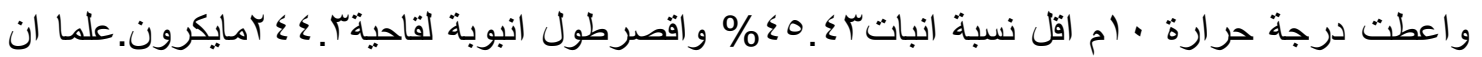

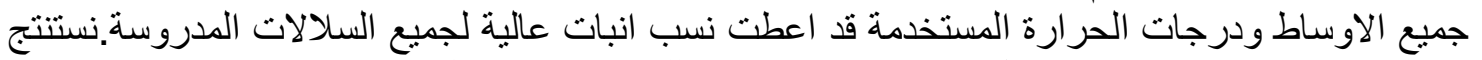

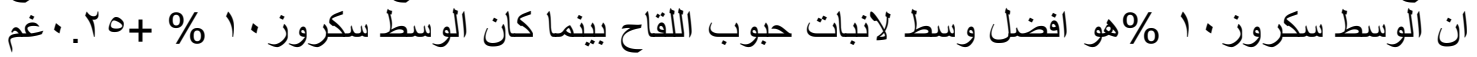

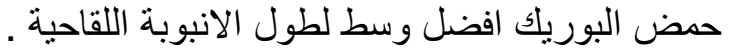

\section{REFERENCES}

Acar,I., and,B.E. AK (2007).An Investigation on pollen germination rate of some selected male trees at ceylanpinar state farm. Dep.of Hort.Fac.of Agr.,Univ.of Harran,63200-Sanliurfa,Turkey

AK ,B.E, A.L Ozguven,.and Y. Nikpeym (1995).An investigation on determining the ability of some Pistacia spp. pollen germination .first international symposium on Pistachio nut,September,20-24,1994,Adana,Turkey,Acta Hort.,419:43-48.

Atli ,H.S., N.,Kaska, and S., Eti (1995)..Selection of male Pistacia spp. Types growing in Gazinatep . $1^{\text {st }}$ international symposium on Pistachio nut,September,20-24,1994,Adana,Turkey,Acta Hort.,419: 319-322

Bots,M.and C. Mariani (2005).Pollen viability in the field .radboud university nijmegn .january-2005.

Calgar,S.\& N., Kaska (2007).Astudy on the supplemintal pollination of pistachios in the mediterranean region $.1^{\text {st }}$ international symposium on pistachio nuts, september,20-24,1944, adana

Crane,J.,H Ford, and C. Daniel (1974) Pollen longevity in pistacia. callifornia Agri.28(11):8-9.

Dafni ,A.and, D. Firmage (2000).Pollen viability and longevity practical, ecological and evolutionary implications. plant systematics and evolution 222:113-132.

Hadj-Hassan, A.(1985) Study of pistachio pollen grain viability at aleppo reagion condition -syria.ACSAD|Th N|N19|1985.syria-demascus.

Johri,B.M.and I.K. Vasil (1961) Physiology of pollen. the botanical review,27(3):325-381.

Mlika,M. (1991). Germination et conservation du pollen de pistacia of GREMPA, June1990, Nimes,France.

Sfendiyaro ,M.E.,Ozeker, A.Misirh, and H .Salam (2006). Determination of pollinator characteristics of different Pistacia sp . depar. of hort. fac. of agri. ege univ.35100 zmir ,turkey P:267-270.

Theriou,K.N.,V.M. Tsikoroglov and K.N. Dimossi-Theriou(1985).Physiological aspects of pistachio pollen germination . rivista ortoflorofruit italy,3:161170.

Ulkummen, L. (1945) Antephstigi uzerinde biology arasharmalar, yuksek ziraat enstitusu dergisi,2(8) :513-539 